\title{
A new system for quantitative evaluation of infant gaze capabilities in a wide visual field
}

\author{
Andrea Pratesi ${ }^{1}$, Francesca Cecchi ${ }^{*}$, Elena Beani ${ }^{2,3}$, Giuseppina Sgandurra ${ }^{2}$, Giovanni Cioni ${ }^{2,4}$, Cecilia Laschi ${ }^{1}$ \\ and Paolo Dario ${ }^{1}$
}

\section{${ }^{*}$ Correspondence:}

f.cecchi@sssup.it

${ }^{1}$ The BioRobotics Institute, Scuola Superiore Sant'Anna, Viale Rinaldo Piaggio 34, 56025 Pontedera, Pisa, Italy Full list of author information is available at the end of the article

\begin{abstract}
Background: The visual assessment of infants poses specific challenges: many techniques that are used on adults are based on the patient's response, and are not suitable for infants. Significant advances in the eye-tracking have made this assessment of infant visual capabilities easier, however, eye-tracking still requires the subject's collaboration, in most cases and thus limiting the application in infant research. Moreover, there is a lack of transferability to clinical practice, and thus it emerges the need for a new tool to measure the paradigms and explore the most common visual competences in a wide visual field. This work presents the design, development and preliminary testing of a new system for measuring infant's gaze in the wide visual field called CareToy C: CareToy for Clinics.
\end{abstract}

Methods: The system is based on a commercial eye tracker (SmartEye) with six cameras running at $60 \mathrm{~Hz}$, suitable for measuring an infant's gaze. In order to stimulate the infant visually and audibly, a mechanical structure has been designed to support five speakers and five screens at a specific distance $(60 \mathrm{~cm})$ and angle: one in the centre, two on the right-hand side and two on the left (at $30^{\circ}$ and $60^{\circ}$ respectively). Different tasks have been designed in order to evaluate the system capability to assess the infant's gaze movements during different conditions (such as gap, overlap or audiovisual paradigms). Nine healthy infants aged 4-10 months were assessed as they performed the visual tasks at random.

Results: We developed a system able to measure infant's gaze in a wide visual field covering a total visual range of $\pm 60^{\circ}$ from the centre with an intermediate evaluation at $\pm 30^{\circ}$. Moreover, the same system, thanks to different integrated software, was able to provide different visual paradigms (as gap, overlap and audio-visual) assessing and comparing different visual and multisensory sub-competencies. The proposed system endowed the integration of a commercial eye-tracker into a purposive setup in a smart and innovative way.

Conclusions: The proposed system is suitable for measuring and evaluating infant's gaze capabilities in a wide visual field, in order to provide quantitative data that can enrich the clinical assessment.

Keywords: Eye-tracker, Gaze-tracking, Infant gaze, Neurodevelopmental bioengineering 


\section{Background}

The assessment of visual capabilities in the first year of life is important for monitoring the development of the infant, but also because if problems occur, they can consequently influence the whole development $[1,2]$. One goal of child health development screening programs is to identify, as early as possible, infants at risk of future visual problems or, more generally, developmental disabilities, in order to make an early intervention possible and incisive [3]. Visual assessments of infants are not easy: many assessment techniques that are used with adults are based on the patient's response thus not suitable for infants since they have such a short attention span. Nevertheless, in recent decades it has become possible to use new assessment methods that have been adapted for the needs of younger patients, that require neither the collaboration of the subject nor special abilities of the examiner; so they are suitable for very young infants, non-verbal or non-collaborative subjects (e.g. patient with mental delay or behavioural problems).

In recent years, eye-tracking has become an increasingly popular tool among researchers in the neurodevelopmental field [4]. Significant advances, particularly in the area of automated eye-tracking, have made this technology much more user-friendly and applicable to human infant populations than ever before [5]. Traditionally, recording eye movements in infants was difficult because of the small field of view required for accurate capture of the pupil. During the test, if the infant steps out from the camera view, data was lost and, of course, infants do not follow instructions to sit still. Other systems such as chinrests and head-mounted optics (cameras and other components) commonly used with adult participants are again impractical for infants since they are more invasive. However, there has been significant progress in the production of lightweight models appropriate for infants. Despite this fast growing interest in infant eye-tracking, the use of this technology has been mostly applied to capturing infant eye movements and gaze patterns when looking at objects or scenes depicted in two dimensions on a computer screen [6-11]. In the studies mentioned above, visual stimulations appear on screens that represent the central visual field and gaze movements are mainly made by the contribution of the eyes. In fact, gaze (the direction of the visual axis in space) is the sum of two contributors: the eye position relative to the head and the head position relative to the space. Over the past few years, eye tracking has been used to examine a variety of perceptual and cognitive phenomena, including categorisation [6, 7, 12-21]. Very few attempts have been developed to assess infant eye tracking in the context of multisensory stimulation in a wide working field covering the peripheral visual field. These studies reported the use of head mounted eye-trackers. These devices require participants to either wear a cap or a band placed on the crown of their head, or wear goggles resting in front of their eyes [5]. Although these devices allowed the participants to navigate in 3D space, several drawbacks such as the invasiveness, the data accuracy in 3D space and the difficulties in detecting eye and head contributions limit their application to infant research. From this analysis, the lack of transferability to clinical practice emerges, and thus the need for a new tool to measure the paradigms and explore the most common visual competences in a wide visual field. This need as a starting point, the aim of this work was to design, develop and test a new system called CareToy C: CareToy for Clinics for quantitatively measuring infant's gaze in a wide visual field $\left(120^{\circ}\right)$ during different conditions (such as gap, overlap or audio-visual paradigms). The innovation was to 
develop a single system for providing different paradigms and to be able to measure and compare infants' eye movements. This work has been inserted into the framework of the EU CareToy project.

\section{Methods}

On the basis of the clinical requirements, the following features have to be taken into account. The system should be able to:

- measure wide range of visual field $\left(120^{\circ}\right)$;

- provide audio-visual stimulation;

- identify five different areas of the visual field for audio-visual stimulation covering $120^{\circ}$;

- measure infants gaze i.e. single contribution of eyes and head respectively;

- represent a non intrusive setup and an ecological approach during clinical trials;

- be adapted according to the infants' needs (aged 3-12 months).

\section{Hardware}

The system consists of an eye-tracker integrated into a customised mechanical structure. The five points are placed in specific positions: one in the centre, two on the right side $\left(30^{\circ}\right.$ and $\left.60^{\circ}\right)$ and two on the left side $\left(30^{\circ}\right.$ and $\left.60^{\circ}\right)$. In order to provide audio-visual stimulations, each point of interest comprises a screen $\left(10.5^{\prime \prime}\right)$ and a speaker. A mechanical structure has been designed in order to fix the screens and the speakers at specific distances and angles (Fig. 1). The screens active area represents our area of interest (AOI), so our stimuli dimensions are $220 \times 129 \mathrm{~mm}$.

We selected USB-VGA converters and an external audio device for managing multimonitors images and sounds signals. Custom software has been developed for the management of the audio-visual stimulations. A dedicated PC has been assigned for the management of these audio-visual stimuli. The external sound card device with six outputs is used to send the sound to the five speakers; the sound card communicates with the PC via the USB port and is connected to the speakers with RCA cables. The desktop is extended to five screens using the USB/VGA converters (Fig. 1).

As far as the eye trackers are concerned, the technological background of the eye tracking technology has been studied to choose the best solutions for our application. In order to obtain a non-intrusive system for measuring the infant gaze, video-oculography (VOG) has been selected. This family of eye trackers is based on corneal-reflection. They assess a video input of the pupil's highlight reflected on the cornea, usually from a light source invisible to humans, in the infrared range of the spectrum. The centre of the pupil and the corneal reflection are tracked in real time, providing information about the participant's point of gaze (POG) on the stimulus [4]. Among the available eye trackers we selected the SmartEye system with six cameras running at $60 \mathrm{~Hz}$ due to the following main features:

Most flexible cameras placement in our setup One of the main advantages of the SmartEye system is its flexibility. The cameras can be positioned independently one from the other, but each camera should be oriented in such way so that the subject's head is 


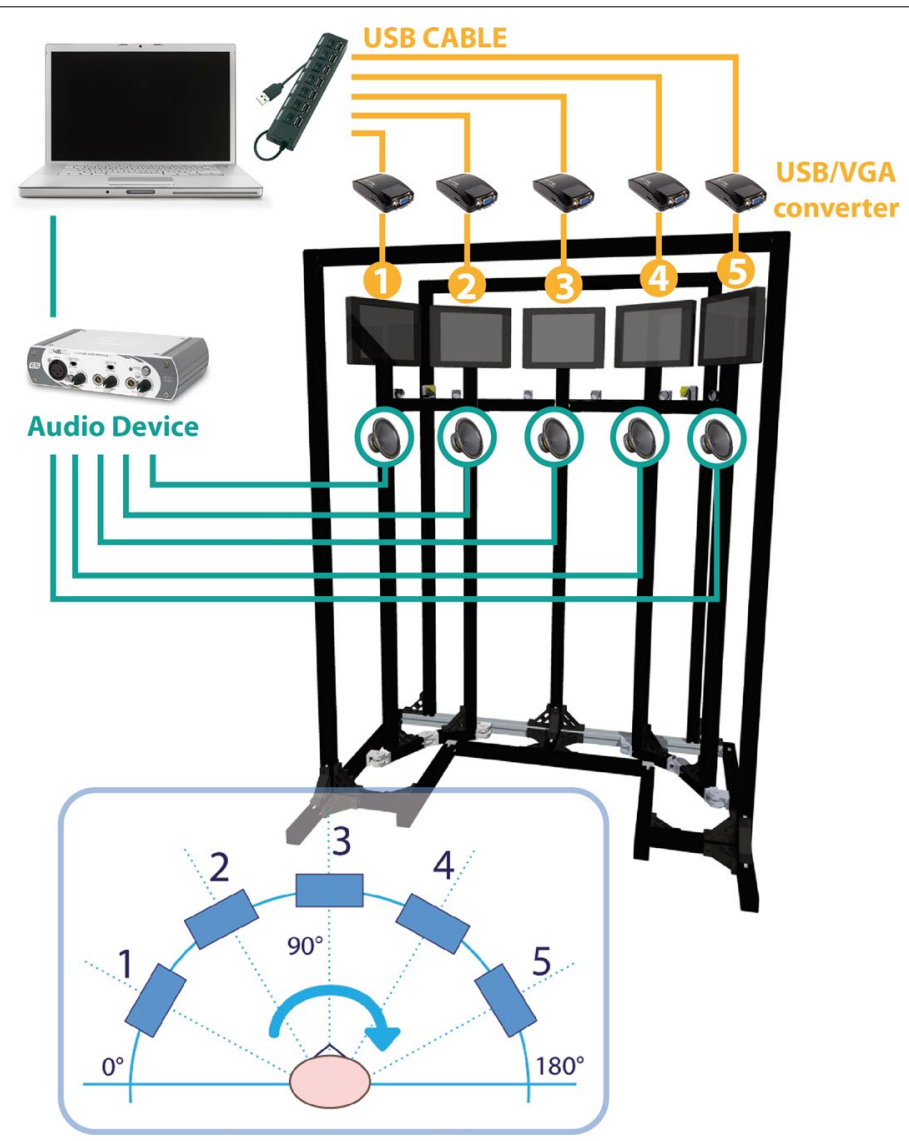

Fig. 1 Schematic system overview. The mechanical custom structure represents the support for the five screens, the five speakers, the six SmartEye cameras running at $60 \mathrm{~Hz}$ and two IR-diodes used for illuminating the face of the subject in order to minimize the effect of varying environmental lighting conditions and for using the reflections of these IR flashes on the cornea ("glints") to find the centre of the eyes. The stimuli management has been obtained using a laptop combined with the audio-video external devices. In the lower part of this overview, it is possible to observe the gaze heading frame of reference

the camera's point of focus. In addition, the number of cameras is not fixed but can be adapted in order to cover the required visual field $\left( \pm 60^{\circ}\right)$.

Gaze and head tracking The system uses IR-diodes to illuminate the face of the subject in order to minimize the effect of varying lighting conditions and use the reflections of these IR flashes on the cornea ("glints") to find the centre of the eyes, rather than other systems where the eye centre is estimated using the head model (Fig. 2). This allows a more accurate identification of gaze direction with fewer errors in the head pose estimation. This feature, i.e. the possibility to measure both eye and head components is extremely important since the visual field proposed in this work is wide and each movement of the infant to reach the audio-visual stimulus is composed of both components. Finally, in order to complete the system and to adjust the setup according to the infant's needs, a seat has been purposely designed allowing for adjustments to the height (the infant's eyes should be at the height of the screen's centre) and the distance from the screen $(\approx 60 \mathrm{~cm})$. In addition, two different accessories complete the seat that allow us to position infants that have not yet reached the stage of torso control, as well as infants that can maintain the sitting posture independently (Fig. 3). 


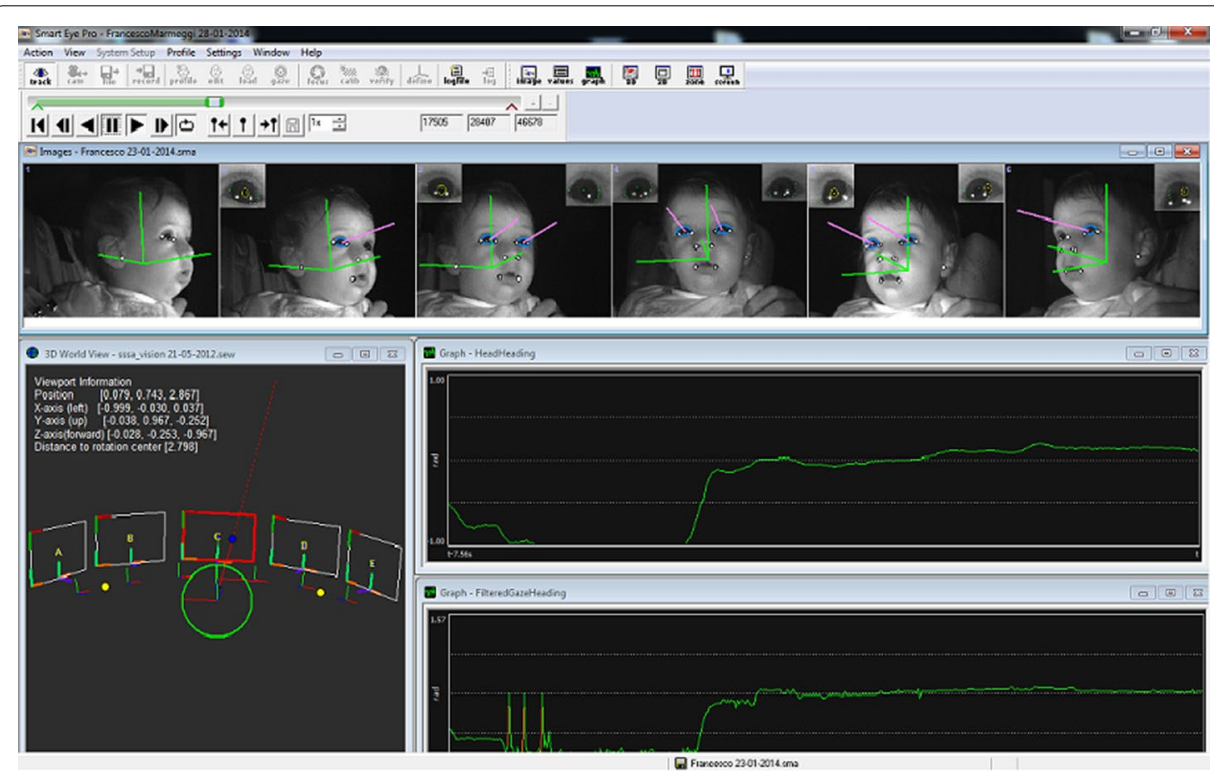

Fig. 2 SmartEye Graphical User Interface (GUI). In the upper bar it is possible see the pink vectors that represent the infant's gaze vector; in the lower window on the left, there is the 3D representation of the external $3 \mathrm{D}$ setup with the visualisation of gaze intersection on a object modelled in the 3D world (i.e. the gaze vector intersects screen n.3) and on the right there are the typical gaze heading and head heading signal profiles

Fig. 3 The final version of the system

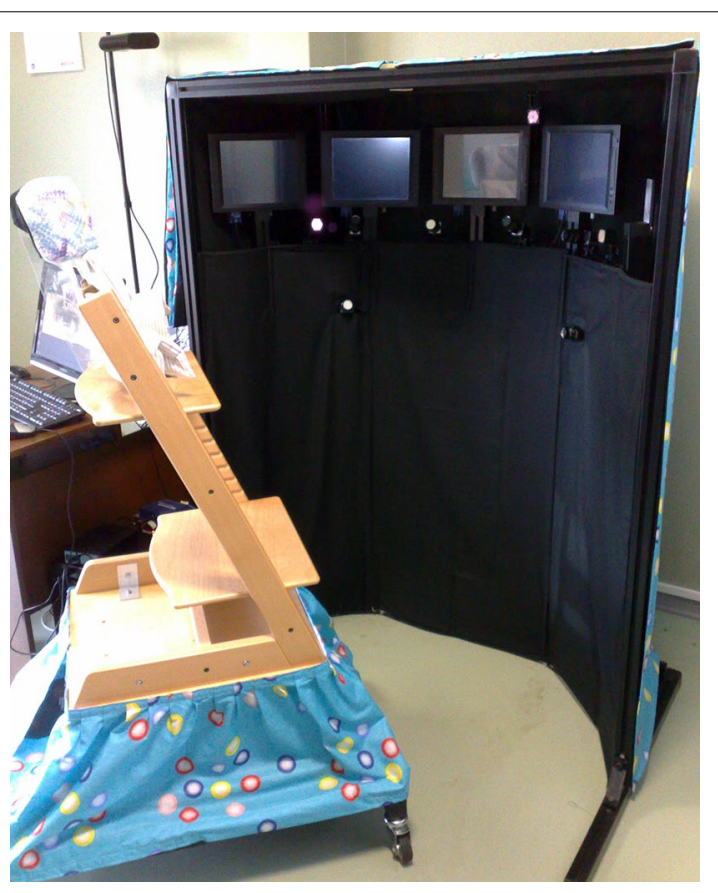

\section{Software}

Purposive software has been developed to create sequences of stimuli (images and sounds) and send them to the screens and speakers. Through the GUI it is possible to set some parameters: (a) the total number of images composing the audio-visual tasks 
sequences; (b) the stimuli (images and sounds) to be presented on each screen and (c) the duration of each stimulus (each sound and image can have a different duration). SmartEye System computer desktop and the PC that manages the presentation of the stimuli are connected to a Local Area Network (LAN) and they synchronize their local time using the Network Time Protocol (NTP).

\section{Experiments}

\section{Clinical background and hypotheses}

Focusing on the assessment of visual attention, the disengagement of attention is the most studied function (engagement, disengagement and attention shift) by using gap and/or overlap paradigms $[22,23]$. In both cases there are two visual stimuli, one on the midline and the other one the on periphery. Both paradigms study the infant's gaze shift from the central to the peripheral stimulus. In the gap condition, the midline stimulus disappears before the peripheral one appears, while it remains present in the overlap paradigm. Clinical studies, without using an eye-tracker, have shown that infants in their first year of life need more time to shift the attention from a central to a peripheral stimulus in the overlap condition [24-26]. Moreover, this effect is more evident in younger infants and decreases with age; this allows us to hypothesize a maturational effect on the gaze shifting competence.

Another interesting aspect is the association between visual and auditory stimuli. When they are combined, the response latencies are fastest with audio-visual targets than the visual targets alone (slower), or the auditory targets (slowest) [27]. All the paradigms have been applied in a limited visual field.

We expected that the purposive setup composing the CareToy $\mathrm{C}$ and the specific software for the management of the audiovisual stimuli would allow the clinicians to develop clinical tasks for the quantitative assessment of visual attention i.e. time and degree of gaze (head and eyes components, separately) in all three (gap, overlap and multisensory) paradigms in a wide field condition.

\section{Experimental paradigm}

The infant is placed at $60 \mathrm{~cm}$ from the screens and the environment is black to avoid distracting factors and to allow the infant to devote their attention to the audio-visual stimuli. The stimuli were chosen in order to be interesting and attractive for infants in their first year of life. There are two series of images for two main categories of age: geometric, circular, concentric or high contrasted pictures (like black/white chessboards, concentric black, white and red images) for younger infants and human faces for older infants. We designed three different tasks to test our hypotheses:

- "attention task" (AT) is the reproduction of the gap paradigm: the stimulus is presented on the central screen; after $3 \mathrm{~s}$ this stimulus disappears and a different stimulus is proposed on one of the peripheral screens $\left(30^{\circ}\right.$ or $60^{\circ}$ on the left/right side, randomly); we expected that the system would be able to measure the shifts of gaze in a non-competitive situation both at $30^{\circ}$ and $60^{\circ}$ on the right and on the left side of visual field. 
- "fixation task" (FT), is the reproduction of the overlap paradigm: the stimulus is presented on the central screen; after $3 \mathrm{~s}$ a lateral stimulus simultaneously appears on the peripheral screen $\left(30^{\circ}\right.$ or $60^{\circ}$ on the left/right side, randomly) in competition with the central one; we expected that the system would be able to measure the shifts of gaze in a competitive situation both at $30^{\circ}$ and $60^{\circ}$ on the right and on the left side of visual field.

- "audio-visual task" (AVT), the stimulus is presented on the central screen; after $3 \mathrm{~s}$ an audio stimulus is presented simultaneously with a visual one on the peripheral screen (i.e. spatially and temporary coherent); we expected that the system would be able to simultaneously produce the audio and visual stimuli and that it would be able to measure the same parameters of AT, detecting the differences in the time parameters between the two tasks (AT and AVT).

The basic sequences of each task were repeated in order to obtain a global duration of 100-120 s. These durations were calibrated based on the average time of visual attention in the first year of life.

\section{Sample}

Nine healthy, born at term, infants ( 4 males, 5 females) with an age range between 4 and 10 months (mean $7 \pm 1.73$ months) were assessed using this system at the IRCCS Fondazione Stella Maris. Each infant performed three different tasks described below (attention, fixation and audio-visual) in a random order. This clinical trial has been approved by the Ethics Committee of Pisa University Hospital and the Tuscan Region Pediatric Ethics Committee (Italy).

\section{Parameters of interest}

Ideally, the profile of the Gaze signal is represented by a ramp divided into three phases (Fig. 4): (a) a fixation phase on the central screen, (b) a gaze movement in the direction of the peripheral screen and (c) a new fixation on the peripheral screen. From this profile, it is possible to identify three main time instants:

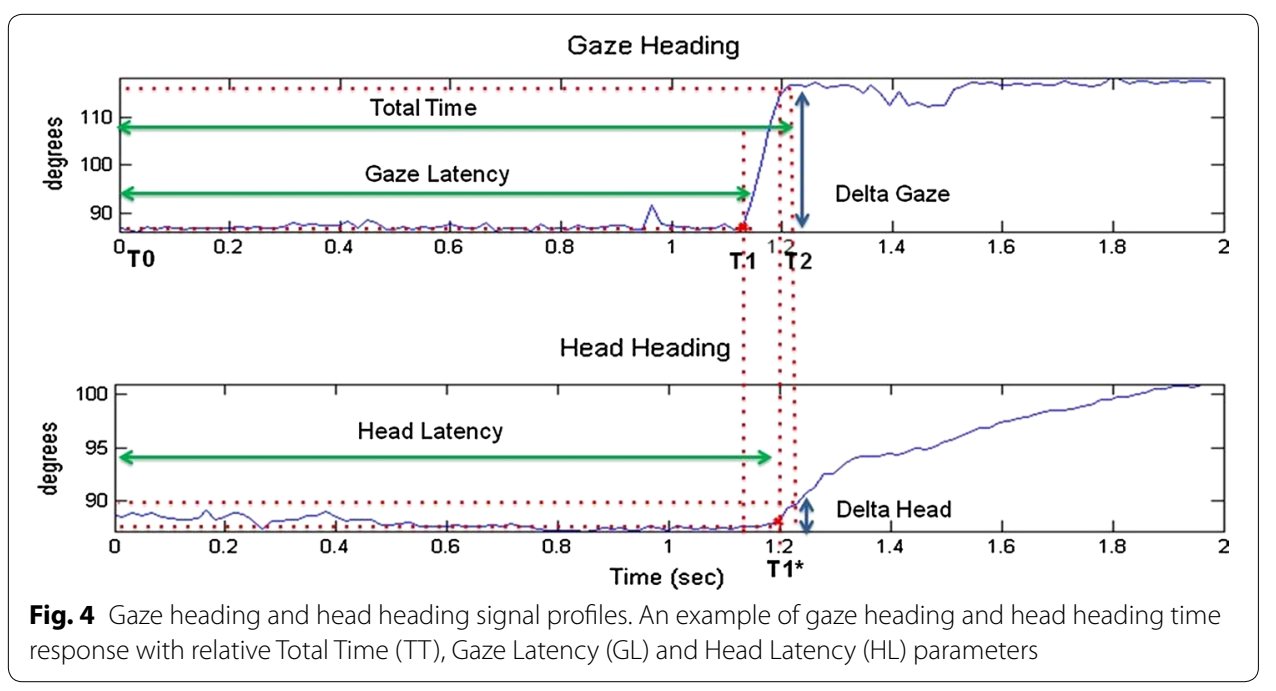


- T0 the stimulus appears on the peripheral screen

- $\mathrm{T} 1$ the gaze begins to move towards the peripheral screen

- T1* the head begins to move towards the peripheral screen

- T2 the gaze intersects the peripheral screen and the movement is completed

Starting from the previous time instants the following time parameters have been selected:

- T0-T1: Gaze latency $(G L)$

- T0-T1*: Head Latency $(H L)$

- T1-T2: Move time (MT)

- T0-T2: Total time (TT)

In addition, we identified delta parameters related to the angular movements:

- Delta Gaze (DG) the angular shift of gaze during MT

- Delta Head (DH) the angular shift of head during T1*-T2 interval

- Delta Eye (DE) the difference between DG and DH

\section{Data selection}

Starting from a more accurate literature evaluation [28], it is important to take into account several factors influencing data quality: different participants' characteristics, level of the operator who performs data acquisition, kind of task used during trial sessions, varying environmental conditions, and last but not least, eye tracker specifications, in terms of: cameras resolution, sharpness of the eye images and calibration procedure. We carefully took care of this last aspect in order to prevent a high data loss rate. Thus, at the beginning of each trial session, we carefully performed a double calibration in order to calibrate both the system and the infant's gaze.

The system calibration was divided into two phases: the first one aimed to adjust the six system cameras to desired positions (small displacements were needed in order to keep the infant's face centred in at least four cameras) and relative camera brightness and focus. This phase consisted of showing attractive and coloured figures combined with different sound stimuli to the infants, sequentially in the five screens. Then, in the second phase, SmartEye Pro 5.9 application automatically detected the current positions and orientation of the cameras, by moving a little chessboard in front of the cameras to calculate their relative position in respect to the whole setup. Starting from these positions, it calculates head and eyes positions.

The purpose of the gaze calibration was to determine the difference between the visual and the optical axis of the eye. We defined five calibration points corresponding to each centre point of the five screens as objects in the syntax of the 3D world model, and the system automatically created calibration points on each screen. This procedure consisted of showing a smile emoticon growing from the centre of each screen combined with a sound stimulus in order to address the infant's gaze to the AOI centres. If the calibration was not successful, e.g. one or more calibration point was missing, we repeated the process at least two times to obtain a satisfactory calibration for all five locations. 
Thanks to SmartEye Pro 5.9 application, it was also possible to quantify the accuracy of the calibration points obtained during recording (Fig. 5). For each point we checked the accuracy and standard deviation of the calibration. The accuracy depended on various parameters such as: (a) distance from the cameras, that we assumed remained fixed $(60 \mathrm{~cm})$; (b) distance and position of the screens (fixed at $60 \mathrm{~cm}$ ); (c) individual differences among infants that we did not include in this study [29]. The calibration was repeated until all calibration points had accuracy lower than $1^{\circ}$.

Moreover, regarding data quality and according to SmartEye technical specifications, it was possible to perform a preliminary data quality evaluation thanks to an important system parameter: Gaze direction quality that is presented in the range [0.0, 1.0]. When the Gaze direction quality is 0 , it means that the system is not tracked correctly and loses the infant's gaze. This parameter expresses a threshold value in order to distinguish reliable data from that to be rejected. Basically it represents a trade-off between recorded data system availability and data accuracy. In order to maintain a reasonable amount of data with sufficient accuracy, we decided that values of gaze direction quality lower than 0.4 would be considered unreliable and consequently rejected.

Furthermore, analysing data in comparison to the gaze heading frame of reference (Fig. 1), we decided to exclude the following cases from the analysis:

- all the trials in which the infant's gaze did not start from the central stimulus

- all the trials in which the gaze shift was outside the working space $\left( \pm 90^{\circ}\right)$.

\section{Data analysis}

Data analysis was devoted to the identification of the time parameters mentioned above and the relative angular movements of head and eyes. T0 can be easily extracted by the

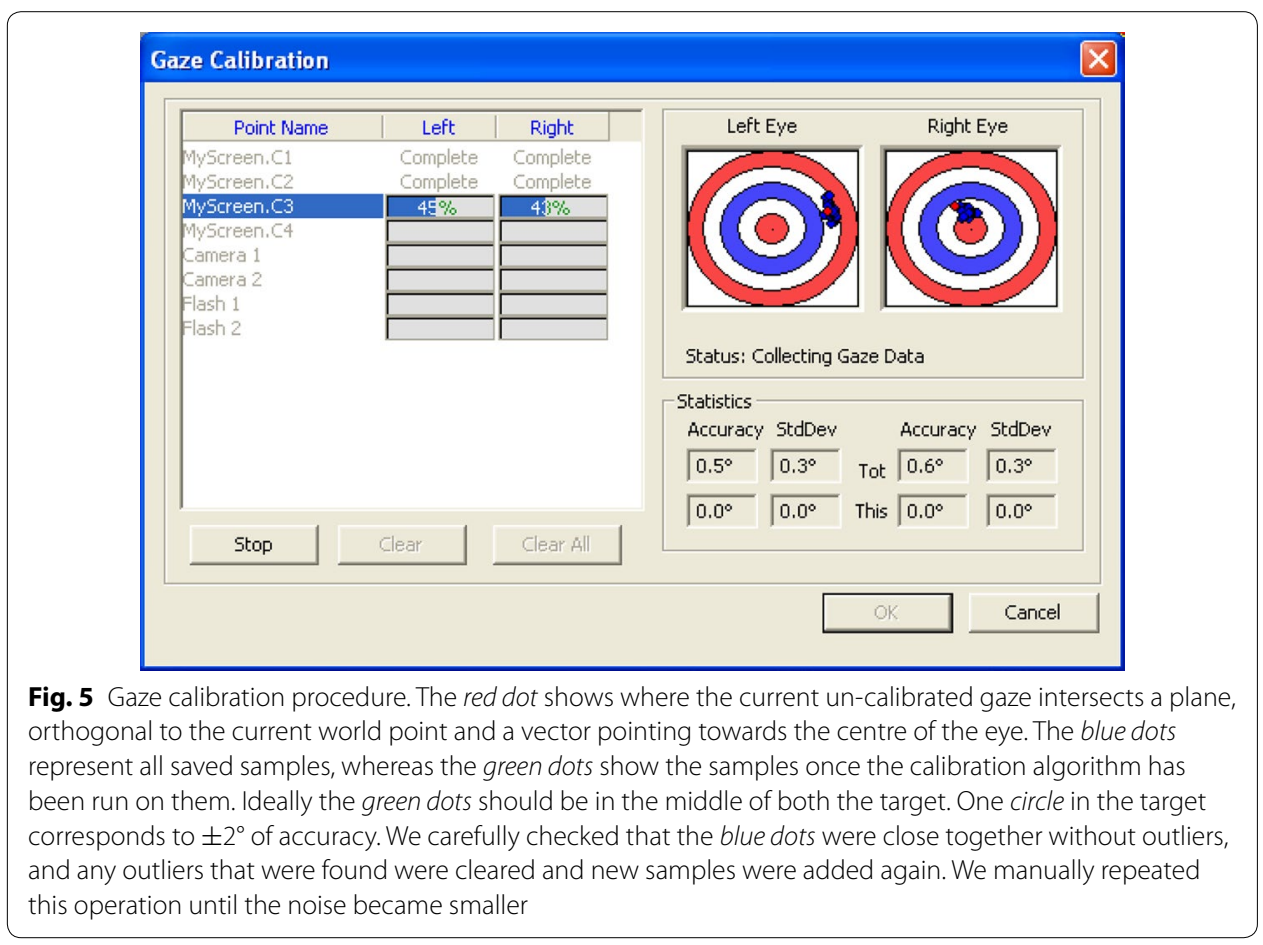


time stamp corresponding to the instant in which the stimulus appears on the peripheral screen given by the stimuli management software. The acceleration vector corresponding to Gaze heading has been used to find T1. When the gaze begins to move, there is a peak in the acceleration signal and this represents the exact parameter of interest (Fig. 6). The acceleration vector has been obtained starting from Gaze vector (second derivative of Gaze parameter).

The adopted strategy was to find all the highest points that represent the peaks and relative time coordinates, and rejecting all gaze movements in the wrong direction. The value of the Gaze heading at the left of the subject is $0^{\circ}, 90^{\circ}$ at the centre and $180^{\circ}$ at the right (see the Fig. 1). If the infant shifted the gaze from screen 3 towards screen 4, we expected a growing gaze angle (for opposite movements, we expected decreasing values). The same procedure was used to find $\mathrm{T} 1$ * from the head heading vector. T2 can be selected from the SmartEye software. Starting from a 3D representation of the external world, the software returns information about the intersection of the gaze with a screen, so it is possible to exactly determine the time in which the gaze intersects each peripheral screen (T2). The spatial parameters of the angular shift are calculated accordingly.

Clinical data was analysed by means of the Statistical Package for Social Sciences (SPSS, version 20.0). Means and standard deviation were calculated and reported in Table 1.

Mann-Whitney $U$ independent sample test was used to analyse the following comparisons in all three tasks: differences between $30^{\circ}$ and $60^{\circ}$ for each parameter and differences between DE and $\mathrm{DH}$ both at $30^{\circ}$ and $60^{\circ}$.

The same non-parametric test was used to compare differences between attention and audio-visual tasks (both $30^{\circ}$ and $60^{\circ}$ ) for each time parameter.

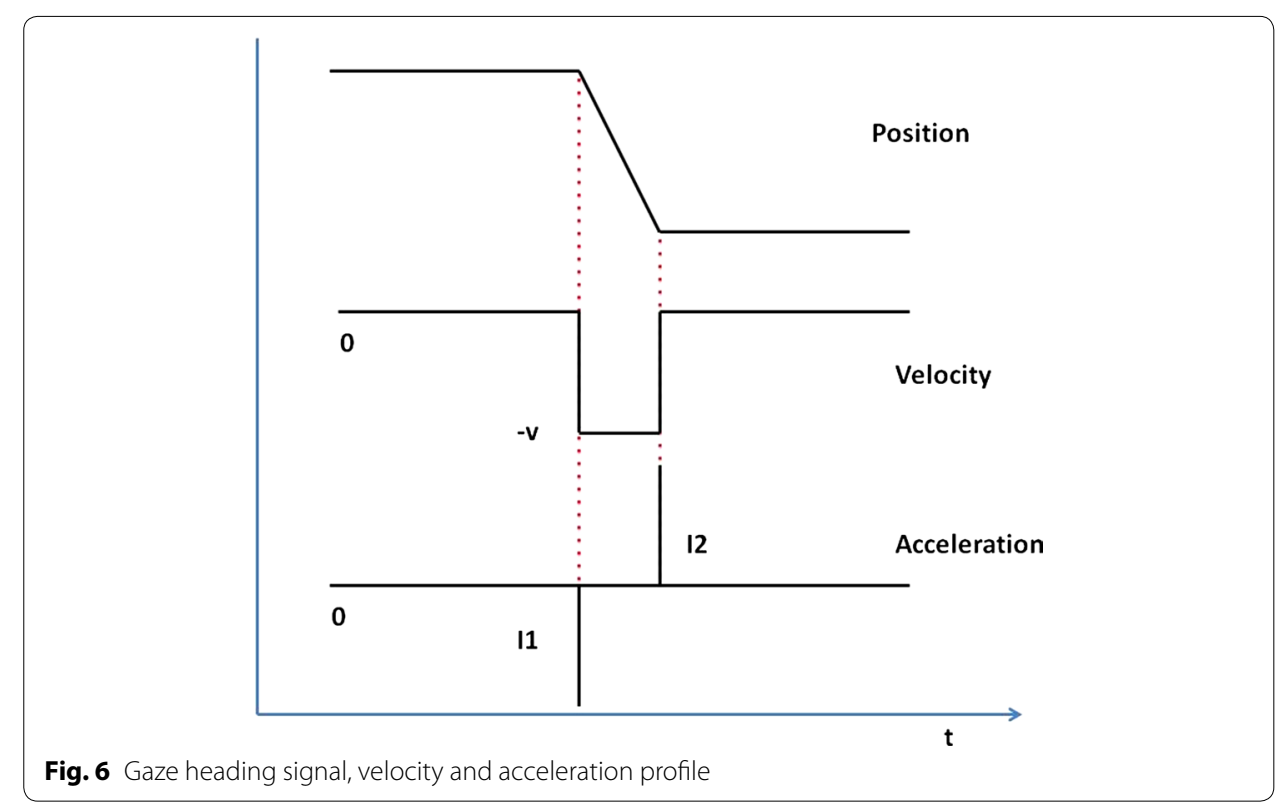


Table 1 Mean values of the main parameters and their relative standard deviation (SD)

\begin{tabular}{|c|c|c|c|c|c|c|}
\hline & \multicolumn{2}{|l|}{ AT } & \multicolumn{2}{|l|}{ FT } & \multicolumn{2}{|l|}{ AVT } \\
\hline & $60^{\circ}$ & $30^{\circ}$ & $60^{\circ}$ & $30^{\circ}$ & $60^{\circ}$ & $30^{\circ}$ \\
\hline \multicolumn{7}{|l|}{ Time } \\
\hline MT (s) & $0.618 \pm 0.347$ & $0.072 \pm 0.017$ & $0.161 \pm 0.036$ & $0.109 \pm 0.102$ & $0.196 \pm 0.133$ & $0.123 \pm 0.165$ \\
\hline $\mathrm{GL}(\mathrm{s})$ & $0.987 \pm 0.492$ & $1.026 \pm 0.322$ & $1.210 \pm 0.687$ & $0.874 \pm 0.299$ & $0.548 \pm 0.199$ & $0.638 \pm 0.241$ \\
\hline $\mathrm{TT}(\mathrm{s})$ & $1.605 \pm 0.181$ & $1.099 \pm 0.326$ & $1.371 \pm 0.683$ & $0.983 \pm 0.287$ & $0.744 \pm 0.096$ & $0.761 \pm 0.109$ \\
\hline \multicolumn{7}{|l|}{ Delta } \\
\hline$D G\left({ }^{\circ}\right)$ & $52.24 \pm 6.60$ & $27.39 \pm 7.08$ & $55.76 \pm 4.67$ & $29.58 \pm 5.05$ & $58.43 \pm 5.66$ & $32.00 \pm 9.09$ \\
\hline $\mathrm{DH}\left({ }^{\circ}\right)$ & $27.72 \pm 8.06$ & $5.64 \pm 5.25$ & $30.24 \pm 14.16$ & $14.84 \pm 9.32$ & $27.11 \pm 8.68$ & $7.79 \pm 6.02$ \\
\hline $\mathrm{DE}\left(\left(^{\circ}\right)\right.$ & $24.51 \pm 3.64$ & $21.75 \pm 8.36$ & $25.52 \pm 14.23$ & $14.74 \pm 10.67$ & $31.32 \pm 7.20$ & $24.21 \pm 4.66$ \\
\hline
\end{tabular}

\section{Results}

Experimental tests gave important results about the capability of the proposed system to track the gaze during the execution of the experimental paradigms. The application of the criteria described in the data selection paragraph brought about a reduction of the total amount of data with a final data loss of approximately $40 \%$.

Figure 7 shows a typical attention task in which an infant orients his/her attention to the periphery (left: from screen 3 to 4 , i.e. $30^{\circ}$, right: from screen 3 to 5 , i.e. $60^{\circ}$ ). More specifically, it shows how transition of the gaze from the central screen (\#3) to the peripheral one (\#4 or \#5) works and data about gaze and head position return information about the contribution of head and eye during the required movement.

The proposed system allows to track the gaze and the head in the whole AOI. A good quality of head position is present when the head is found and tracked in at least two cameras of the eye-tracker. Thank to the presence of six cameras, the head is almost
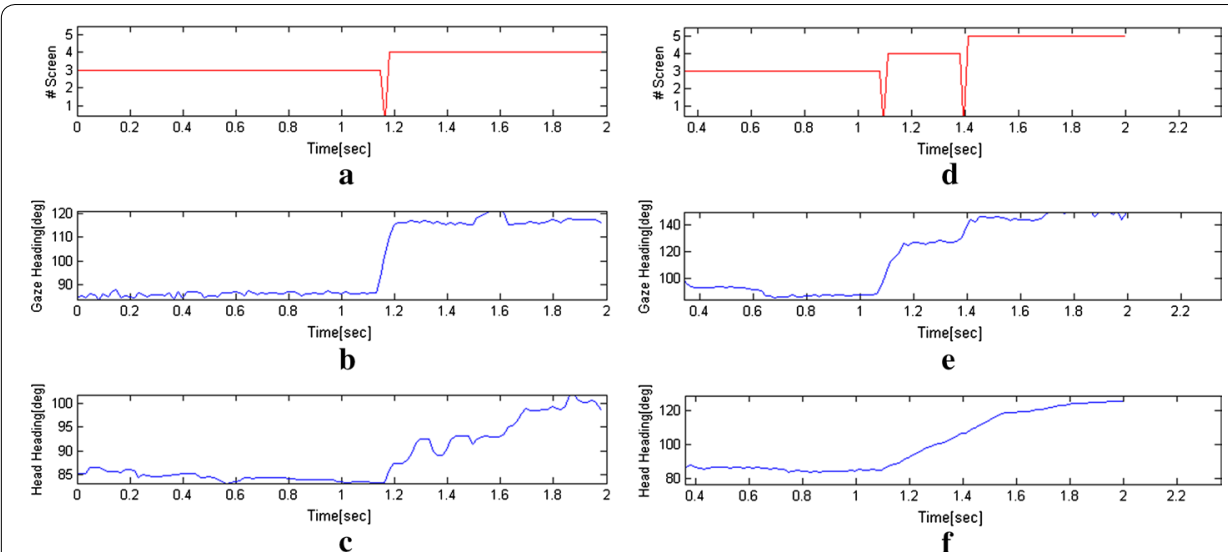

$\mathbf{e}$

Fig. 7 Examples of attention task: a results of SmartEye analysis of intersection between the gaze with the screens. During the transition from screen \#3 to \#4, the gaze passes through the space between the two screens thus it does not intersect one of the $\mathrm{AOI}$ and the system returns zero value, $\mathbf{b}$ gaze heading during the transition from screen \#3 to screen \#4, c head heading during the transition from screen \#3 to screen \#4, d results of SmartEye analysis of intersection between the gaze with the peripheral screens. In this case the system returns zero value when the gaze is between screen 3 and 4 and between 4 and 5, e gaze heading during the transition from screen \#3 to screen \#5, f head heading during the transition from screen \#3 to screen \#5 
visible in two cameras during the entire duration of the tasks. This particular feature reduces the data loss and inaccuracies that might result from nonoptimal head orientations revealed in other works [30].

It is worth to mention also noise issue, the SmartEye system gives the possibility of setting parameters that affects the gaze output values called 'Filtered' and consequently the signal noise (e.g. saccade and pupil filters). In details, it is possible to specify at which angle an eye movement will be classified as a saccade and how long the fixation filter should be. In addition, the diameter of the pupil does not change very rapidly; temporal filtering is therefore used successfully to reduce the amount of noise.

From data analysis of the three tasks, it is possible to observe relevant information presented in the following sections about system capabilities to measure infant behaviour in all of the three paradigms. Results are presented on the basis of the defined parameters.

\section{Time parameters}

In all the three tasks, MT values are lower when stimuli are presented at $30^{\circ}$ with compared to $60^{\circ}$ (AT: $\mathrm{p}=0.001$, FT: $\mathrm{p}=0.003$, AVT: $\mathrm{p}=0.015$ ) (Fig. 8).

Attention tasks present similar values of GL at $30^{\circ}$ and $60^{\circ}(\mathrm{p}>0.05)$ as in audio-visual ones ( $p>0.05)$, while in the fixation task it is possible to observe slightly higher and more variable values at $60^{\circ}$ compared to $30^{\circ}(\mathrm{p}>0.05)$. It is worth saying that $60^{\circ}$ values present high variability (Fig. 8).

Since TT is the sum of the previous values, we can observe that in the attention task, TT values are higher at $60^{\circ}$ compared to $30^{\circ}(\mathrm{p}=0.003)$. The same trend is present in the fixation task even if it is not significant $(\mathrm{p}>0.05)$. This may be due to the high variability of the values at $60^{\circ}$. On the contrary, the audio-visual task presents similar values of TT at $30^{\circ}$ and $60^{\circ}(\mathrm{p}>0.05)$ (Fig. 8).

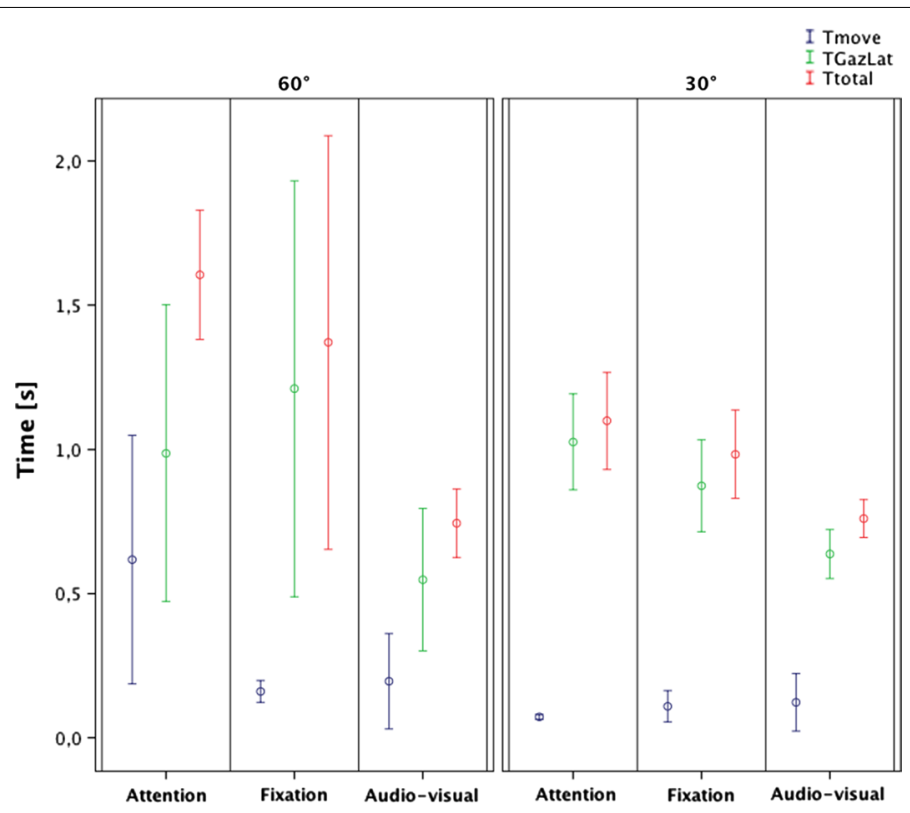

Fig. 8 Time parameters results 


\section{Comparison of time parameters between attention and audio-visual tasks}

In the audio-visual task, at $30^{\circ} \mathrm{TT}$ and GL are largely lower than in the attention (TT: $\mathrm{p}=0.016$, GL: $\mathrm{p}=0.008$ ), while MT presents the same value ( $\mathrm{p}>0.05)$. At $60^{\circ}$, TT is largely lower in the audio-visual task $(\mathrm{p}<0.009)$ while the other two parameters are slightly lower (MT: $\mathrm{p}=0.047, \mathrm{GL}: \mathrm{p}>0.05$ ).

\section{Delta parameters}

By comparing values between $60^{\circ}$ and $30^{\circ}$, in all three tasks, DG and DH values at $60^{\circ}$ are significantly higher than at $30^{\circ}$ (DG: AT p =0.001, FT p < 0.001, AVT p = 0.003; DH: AT $\mathrm{p}=0.001$, FT $\mathrm{p}=0.008$, AVT $\mathrm{p}=0.004)$. DE values are similar in attention and fixation tasks ( $p>0.05)$ and also in the audio-visual even if the difference between the two values are limited $(\mathrm{p}=0.04)$. In the attention and audio-visual tasks, at $30^{\circ}$, it is possible to observe that DE values are higher than DH values (AT: $p<0.001$, AVT: $p<0.001$ ) while they are similar in fixation task ( $\mathrm{p}>0.05)$. At $60^{\circ}, \mathrm{DE}$ values and $\mathrm{DH}$ values are similar in all the three tasks ( $\mathrm{p}>0.05)$ (Fig. 9).

\section{Discussion}

In the last decade there has been an explosion of research using eye tracking with infants thanks to the evolution of the technological solution. However, automatic eye tracking presents several challenges such as the need for a good calibration procedure, the need for a purposeful experimental paradigm for infants and the difficulties of data processing [31].

In this work, the technological challenge was to build a system able to measure an infant's gaze in a wide visual field covering a total visual range of $\pm 60^{\circ}$ from the centre with an intermediate evaluation at $\pm 30^{\circ}$. Moreover, the same system, thanks to different integrated software, was able to provide different visual paradigms (as gap, overlap and

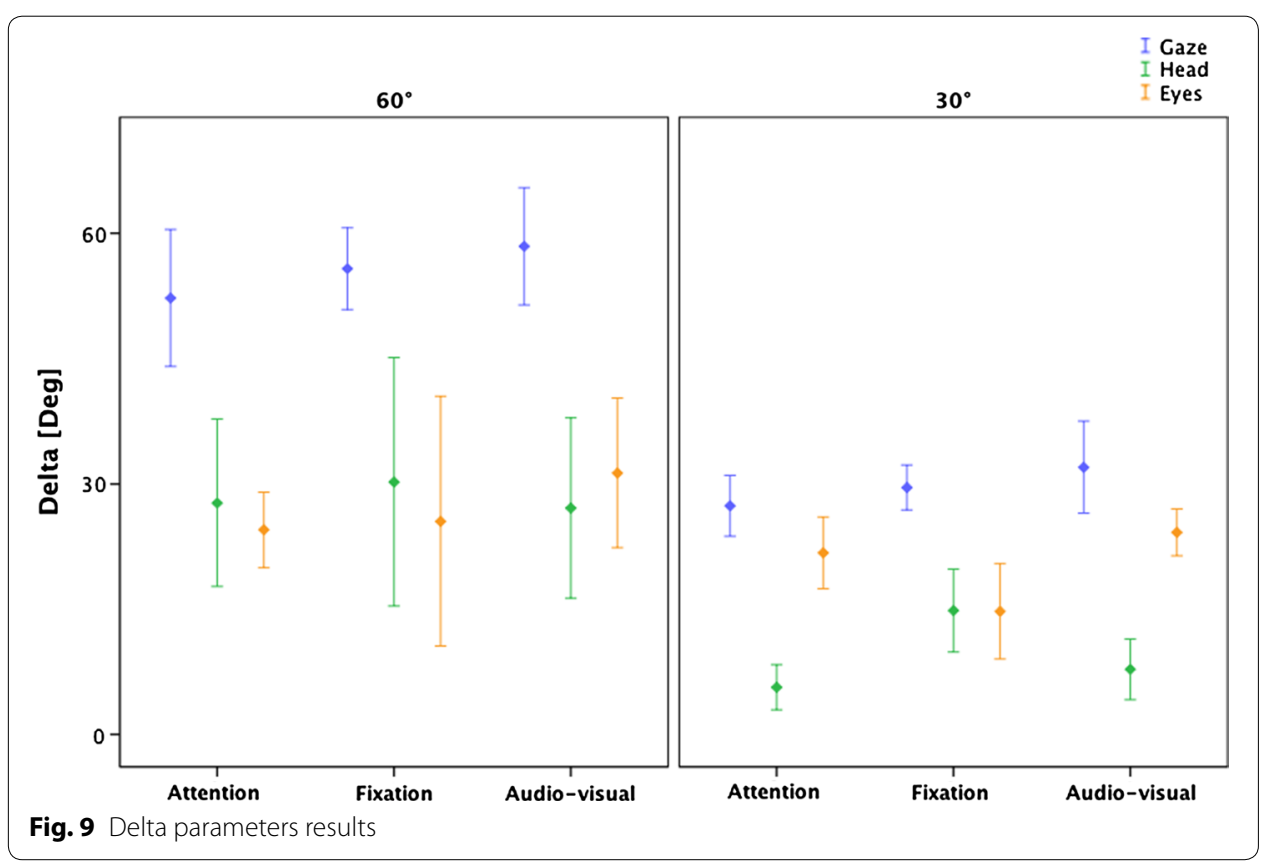


multisensory) assessing and comparing different visual sub-competencies. The proposed system endowed the integration of a commercial eye-tracker into a purposive setup in a smart and innovative way. The calibration procedures of the system and the infant's gaze allowed us to obtain reliable data with an accuracy less than $1^{\circ}$.

One encouraging result is represented by the feasibility of the assessment. The infants performed the three tasks and the system acquired their quantitative data.

The infants maintained the sitting posture with ease and the environment allowed the infant to only pay attention to the stimuli, avoiding the distracting factors. Also the duration of the experiment was well calibrated: all the infants managed to successfully complete all the three tasks.

The results provided two kinds of data: delta and time values. The system allowed us to obtain detailed information of each task about the gaze shift and visual attention in terms of distance and movement of the head and eyes as well as duration and latency. Additionally, it was possible to make comparisons in each task between $30^{\circ}$ and $60^{\circ}$ and also between tasks (AT vs AUV).

Compared with previous studies, the expectations and the past findings have been obtained, and it has been possible to study the behaviour at two distances from the centre of the visual field: 30 and 60 visual degrees.

Even if the small sample cannot give information and generalisations about visual functioning, some interesting findings are promising for the use of CareToy $\mathrm{C}$ in the clinical practice. The main strength is that the same system can measure different abilities in the same infant and across infants within different ages, exploring a wide visual field and distinguishing the eye and the head component contributions to the gaze. An interesting finding about time parameters is that the system was able to discriminate between different behaviour in presence or absence of sound stimuli, confirming the literature [27] that the speed is higher in the presence of sound. In particular, the CareToy $\mathrm{C}$ system detected that with the presence of sound the TT values are similar at $30^{\circ}$ and $60^{\circ}$. Comparing audio-visual (AVT) and attention (AT) tasks (it is worth underlining that those tasks are identical except for the spatial associations of the visual stimulus with the sound) the system has shown, in the AVT a faster visual response at $30^{\circ}$ with lower values of the GL and at $60^{\circ}$ lower values not only GL but also of MT. Another interesting result is that the system highlighted higher variability of the TT, related to the GL component in the overlap condition, probably due to the presence of a competitive stimulus (fixation task) which makes the task challenging at $60^{\circ}$. Regarding delta parameters, the analysis of DG confirms the reliability of the CareToy $C$ system because, as expected, the values are in the range of $30^{\circ}$ or $60^{\circ}$ demonstrating that a larger movement is necessary to reach the more peripheral areas of the visual field. Moreover, the system is able to quantitatively distinguish eye and head components. An interesting finding, to be confirmed in a larger group of infants, is that stimuli at $30^{\circ}$ seem to be visually detected using mainly eye movements, while for larger movements $\left(60^{\circ}\right)$ a head compensatory movement is required. This behaviour is evident in attention and audio-visual tasks, instead of fixation task, in which the head contribution is already evident at $30^{\circ}$; this could be due to the presence of a competitive stimulus and the relative difficulty in the gaze disengagement. Further studies on the different strategies at $30^{\circ}$ and $60^{\circ}$ across 
different ages could be very interesting for new hypotheses of visual development and applications (e.g. new treatment strategies).

This study presents some limitations. First of all, the data loss remains a critical point; future work will investigate algorithms for improving data quality [32] and the possibility to trigger the stimuli on the screens on the basis of the current position of the gaze obtained with the eye-tracker. Furthermore, the sample size of this study was quite small, but it allows us to demonstrate the feasibility of the purpose. An interesting future development could be to test a wider sample in order to obtain quantitative about the general development of visual perception in infancy.

\section{Conclusion}

In conclusion, this new system is a suitable tool for measuring and evaluating infants' gaze capabilities in a wide visual field in order to provide quantitative data that can enrich the clinical assessment, e.g. for objectively evaluating changes after a treatment [33].

\section{Abbreviations}

AOI: area of interest; VOG: video-oculography; POG: point of gaze; GUl: Graphical User Interface; LAN: Local Area Network; NTP: Network Time Protocol; AT: attention task; FT: fixation task; AVT: audio-visual task; GL: gaze latency; HL: head latency; MT: move time; TT: total time; DG: Delta Gaze; DH: Delta Head; DE: Delta Eye.

\section{Authors' contributions}

PD, CL and GC are the chief investigators and together with AP, FC, EB and GS designed and established this research study. AP and FC were responsible for the design and development of the presented system, with the supervision of PD and CL. Additionally AP and FC performed data analysis and extrapolation of the parameters of interest. EB and GS were responsible for design of the experimental paradigm and clinical interpretation of results. AP and EB drafted the manuscript. All authors read and approved the final manuscript.

\section{Authors' information}

AP is a biomedical engineer and assistant researcher at The BioRobotics Institute of the Scuola Superiore Sant'Anna, a research centre in the field of advanced robotics and biomedical engineering, in Pontedera, Pisa. FC is a post-doc researcher at The BioRobotics Institute of the Scuola Superiore Sant'Anna, in the fields of neurodevelopmental engineering and educational robotics, and the project manager of the EU project CareToy. EB is a physical therapist at IRCCS Fondazione Stella Maris in Pisa, which is a research hospital for children and adolescents with neurological and psychiatric disorders, located in Calambrone, Pisa and a PhD student in Neuroscience at University of Florence. GS is a specialist in child neuropsychiatry and a researcher at IRCCS Fondazione Stella Maris in Pisa. GC is a specialist in child neuropsychiatry, a full professor of Child Neuropsychiatry at University of Pisa and the Scientific Director of IRCCS Fondazione Stella Maris. CL is a full professor of BioRobotics (Italian academic sector of Biomedical Engineering) at the Scuola Superiore Sant'Anna. PD is a full professor of Biomedical Engineering, the coordinator of the PhD Program in BioRobotics and the Director of The BioRobotics Institute of the Scuola Superiore Sant'Anna.

\section{Author details}

${ }^{1}$ The BioRobotics Institute, Scuola Superiore Sant'Anna, Viale Rinaldo Piaggio 34, 56025 Pontedera, Pisa, Italy. ${ }^{2}$ Department of Developmental Neuroscience, IRCCS Fondazione Stella Maris, Viale del Tirreno 331, 56128 Calambrone, Pisa, Italy. ${ }^{3}$ Department of Neuroscience, Psychology, Drug Research and Child Health, University of Florence, Florence, Italy. ${ }^{4}$ Department of Clinical and Experimental Medicine, University of Pisa, Via Roma 67, 56125 Pisa, Italy.

\section{Acknowledgements}

We would like to sincerely thank all of the infants and their families who participated in this research. We also thank MD Francesca Tinelli for her support in the design of the clinical protocols and Ilde Barbieri for her contribution in the development of the software for managing the audio-visual stimuli. A special thank to Georgina Holt for reviewing the English of the article.

\section{Funding}

This work was funded by the European Union Collaborative Project CareToy Grant ICT-2011.5.1-287932, and it was partially funded by the Italian Ministry of Health (GR-2011-02350053).

\section{Compliance with ethical guidelines}

\section{Competing interests}

The authors declare that they have no competing interests.

\section{Consent}

Detailed information about the protocol were provided to the families and, IF parental written consent was obtained, infants were enrolled. 
Received: 3 March 2015 Accepted: 12 August 2015

Published online: 07 September 2015

\section{References}

1. Dubowitz LM, Dubowitz V, Morante A. Visual function in the newborn: a study of preterm and full-term infants. Brain Dev. 1980;2:15-29.

2. Madan A, Jan JE, Good WV. Visual development in preterm infants. Dev Med Child Neurol. 2005;47:276-80.

3. Morante A, Dubowitz LM, Leven M, Dubowitz V. The development of visual function in normal and neurologically abnormal preterm and fullterm infants. Dev Med Child Neurol. 1982;24:771-84

4. Gredebäck G, Johnson S, von Hofsten C. Eye tracking in infancy research. Dev Neuropsychol. 2010;35:1-19.

5. Corbetta D, Guan Y, Williams JL. Infant eye-tracking in the context of goal-directed actions. Infancy. 2012;17:102-25.

6. Quinn PC, Doran MM, Reiss JE, Hoffman JE. Time course of visual attention in infant categorization of cats versus dogs: evidence for a head bias as revealed through eye tracking. Child Dev. 2009;80:151-61.

7. Johnson SP, Amso D, Slemmer JA. Development of object concepts in infancy: evidence for early learning in an eyetracking paradigm. Proc Natl Acad Sci. 2003;100:10568-73.

8. Farzin F, Rivera SM, Whitney D. Spatial resolution of conscious visual perception in infants. Psychol Sci. 2010:21:1502-9.

9. Farzin F, Rivera SM, Whitney D. Time crawls: the temporal resolution of infants' visual attention. Psychol Sci. 2011;22:1004-10.

10. Gredebäck G, von Hofsten C. Infants' evolving representations of object motion during occlusion: a longitudinal study of 6- to 12-month-old infants. Infancy. 2004;6:165-84.

11. Johnson SP, Slemmer JA, Amso D. Where infants look determines how they see: eye movements and object perception performance in 3-month-olds. Infancy. 2004;6:185-201.

12. Johnson SP, Shuwairi SM. Learning and memory facilitate predictive tracking in 4-month-olds. J Exp Child Psychol. 2009;102:122-30.

13. Kirkham NZ, Slemmer JA, Richardson DC, Johnson SP. Location, location, location: development of spatiotemporal sequence learning in infancy. Child Dev. 2007;78:1559-71.

14. Richmond J, Nelson CA. Relational memory during infancy: evidence from eye tracking. Dev Sci. 2009;12:549-56.

15. Amso D, Johnson SP. Learning by selection: visual search and object perception in young infants. Dev Psychol. 2006:42:1236-45.

16. Von Hofsten C, Kochukhova O, Rosander K. Predictive tracking over occlusions by 4-month-old infants. Dev Sci. 2007;10:625-40.

17. Johnson SP, Davidow J, Hall-Haro C, Frank MC. Development of perceptual completion originates in information acquisition. Dev Psychol. 2008;44:1214-24.

18. Kato M, de Wit TCJ, Stasiewicz D, von Hofsten C. Sensitivity to second-order motion in 10-month-olds. Vis Res. 2008;48:1187-95.

19. Frank MC, Vul E, Johnson SP. Development of infants' attention to faces during the first year. Cognition. 2009;110:160-70.

20. Turati C, Valenza E, Leo I, Simion F. Three-month-olds' visual preference for faces and its underlying visual processing mechanisms. J Exp Child Psychol. 2005;90:255-73.

21. Falck-Ytter T, Gredebäck G, von Hofsten C. Infants predict other people's action goals. Nat Neurosci. 2006;9:878-9.

22. Blaga OM, Colombo J. Visual processing and infant ocular latencies in the overlap paradigm. Dev Psychol. 2006:42:1069-76.

23. Atkinson J, Hood B, Wattam-Bell J, Braddick O. Changes in infants' ability to switch visual attention the first three months of life. Perception. 1992;21:643-53.

24. Matsuzawa M. Development of saccade target selection in infants. Percept Mot Skills. 2001;93:115-23.

25. Macfarlane A, Harris P, Barnes I. Central and peripheral vision in early infancy. J Exp Child Psychol. 1976;21:532-8.

26. Guzzetta A, Cioni G, Cowan F, Mercuri E. Visual disorders in children with brain lesions: 1. Maturation of visual function in infants with neonatal brain lesions: correlation with neuroimaging. Eur J Paediatr Neurol. 2001;5:107-14

27. Neil PA, Chee-Ruiter C, Scheier C, Lewkowicz DJ, Shimojo S. Development of multisensory spatial integration and perception in humans. Dev Sci. 2006;9:454-64.

28. Holmqvist K, Nyström M, Mulvey F. Eye tracker data quality: what it is and how to measure it. In: Proceedings of the symposium on eye tracking research and applications (ETRA'12). New York: ACM; 2012. p. 45-52.

29. Leppänen JM, Forssman L, Kaatiala J, Yrttiaho S, Wass S. Widely applicable MATLAB routines for automated analysis of saccadic reaction times. Behav Res Methods. 2014:47:538-48.

30. Hessels RS, Cornelissen THW, Kemner C, Hooge ITC. Qualitative tests of remote eyetracker recovery and performance during head rotation. Behav Res Methods 2014:1-12. doi:10.3758/s13428-014-0507-6

31. Oakes LM. Advances in eye tracking in infancy research. Infancy. 2012;17:1-8.

32. Wass SV, Forssman L, Leppänen J. Robustness and precision: how data quality may influence key dependent variables in infant eye-tracker analyses. Infancy. 2014;19:427-60.

33. Sgandurra G, Bartalena L, Cioni G, Greisen G, Herskind A, Inguaggiato E, Lorentzen J, Nielsen JB, Sicola E. Homebased, early intervention with mechatronic toys for preterm infants at risk of neurodevelopmental disorders (CARETOY): a RCT protocol. BMC Pediatr. 2014;14:268 